\title{
Intermittent regurgitation caused by incomplete leaflet closure of the Medtronic ADVANTAGE bileaflet heart valve: Analysis of the underlying mechanism
}

\author{
Walter B. Eichinger, MD, Ina Hettich, MD, Sabine Bleiziffer, MD, Ralf Günzinger, MD, \\ Andrea Hutter, MD, Robert Bauernschmitt, MD, and Ruediger Lange, MD
}

\begin{abstract}
Objective: Clinical echocardiographic assessments of the Medtronic ADVANTAGE (Medtronic Inc, Minneapolis, Minn) prosthesis in the aortic position revealed a phenomenon identified as "intermittent regurgitation." An in vitro investigation was initiated to identify the underlying mechanism.
\end{abstract}

\begin{abstract}
Methods: In a pulse duplicator environment, 6 ADVANTAGE size 23 aortic valves were analyzed. Leaflet motion and flow through the valves were documented using echocardiography with color Doppler flow, digital high speed imaging, and flow meter assessment.

Results: Intermittent regurgitation could be reproduced in all 6 of the tested valves within limited ranges of flow, pressure, and valve orientation. By virtue of high-speed imaging, the mechanism underlying intermittent regurgitation was identified. During intermittent regurgitation, the leading edge of the second-to-close leaflet makes contact with the chamfer on the leading edge of the first-to-close leaflet. The fluid closing forces working on the first-to-close leaflet prevent it from shifting back so that the leading edge of the second-to-close leaflet remains positioned against the chamfer of the first-to-close leaflet. In this position, the major radius of the second-to-close leaflet does not reach the housing's major radius. Therefore, a crescent-shaped gap remains between the leaflet tip of the second-to-close leaflet and the housing major radius during all or part of diastole. The regurgitant fraction can increase from a normal range of $6 \%$ to $25 \%$ during an intermittent regurgitation beat.
\end{abstract}

Conclusions: In vitro intermittent regurgitation can be induced in the size 23 aortic ADVANTAGE valve under a limited range of conditions. To avoid possible misinterpretations, the phenomenon must be known in detail by all physicians dealing with patients with an ADVANTAGE valve. (J Thorac Cardiovasc Surg 2010;140:611-6)

In 2006 the first report ${ }^{1,2}$ was published of a phenomenon identified as "intermittent regurgitation", (IR), which occurred after implantation of a Medtronic ADVANTAGE (Medtronic Inc, Minneapolis, Minn) bileaflet heart valve prosthesis in the aortic position. Since then, 3 reoperations have been performed in different centers in patients whose aortic valve prosthesis showed IR only because the phenomenon had been misinterpreted as potential valve thrombosis. ${ }^{3}$ After learning of the first 2 reoperations, Medtronic Inc, the manufacturer of the ADVANTAGE valve, communicated the IR phenomenon worldwide to implanters of the ADVAN-

\footnotetext{
From the German Heart Center Munich, Department of Cardiovascular Surgery, Munich, Germany.

Funding: In vitro measurements were performed by Medtronic Inc, Minneapolis, Minnesota.

Disclosures: Walter Echinger reports consulting/lecture fees and grant support from Medtronic and St. Jude. Andrea Hutter reports lecture fees and grant support from Edwards. Rudiger Lange reports consulting fees and grant support from Medtronic and Edwards.

The German Heart Center is part of the investigating institutions in the ADVANTAGE trial initiated by Medtronic Inc.

Received for publication April 7, 2009; revisions received Aug 28, 2009; accepted for publication Nov 1, 2009; available ahead of print Feb 1, 2010.

Address for reprints: Ina Hettich, MD, German Heart Center, Clinic of Cardiovascular Surgery, Lazarettstraße 36, 80636 Munich, Germany (E-mail: hettich@dhm.mhn.de). $0022-5223 / \$ 36.00$

Copyright (c) 2010 by The American Association for Thoracic Surgery

doi:10.1016/j.jtcvs.2009.11.001
}

TAGE valve. Because the mechanism of IR was not clear, the German Heart Center Munich and Medtronic Inc initiated an in vitro investigation to detect the underlying mechanism.

\section{MATERIALS AND METHODS \\ In Vivo Findings in Patients Presenting With Intermittent Regurgitation}

All bileaflet mechanical valves exhibit some minor regurgitation at the pivot points. ${ }^{4}$ The pivot region is designed to allow washing jets to reduce the risk of thrombus formation. In circumstances in which the echo windows are of sufficient quality to permit good diagnostic imaging, this trivial regurgitation is seen in every diastole by color Doppler flow imaging as 2 small jets not exceeding the left ventricular outflow tract, homogeneous in color, reflecting its low momentum. ${ }^{5}$

In contrast, the clinical echo findings in IR were intermittent, single-beat, mild-to-moderate regurgitant jets during diastole. Those jets were different in extension and grade of insufficiency compared with the expected washing jets in bileaflet valves. The phenomenon appeared in irregular intervals and was reproducible in most of the consecutive echo investigations performed on individual patients.

Fluoroscopy of the patients who exhibited echocardiographic signs of IR revealed intermittent incomplete closure of the valve leaflets, which could involve either 1 of the 2 leaflets in the same prosthesis. Simultaneous direct blood pressure measurement in the ascending aorta approximately $2 \mathrm{~cm}$ distally to the prosthesis showed a diastolic pressure decrease during each IR beat. ${ }^{1,2}$

\section{Medtronic ADVANTAGE Prosthetic Heart Valve}

The Medtronic ADVANTAGE prosthesis is a bileaflet mechanical heart valve. The design consists of 2 pyrolytic carbon-coated leaflets constrained 


\section{Abbreviation and Acronym \\ $\mathrm{IR}=$ intermittent regurgitation}

within a pyrolytic carbon-coated housing. A rotatable, fabric sewing ring is affixed to the valve by a titanium insert ring that is located below the level of the carbon housing. A new asymmetric, multilevel butterfly hinge mechanism is realized in this prosthesis, the leaflet opening angle is 86 degrees, and the closing angle is 22 degrees. The ADVANTAGE valve has been in clinical use since 1999 with approximately 11,000 patients receiving implants worldwide up to the present (Medtronic Inc, personal communication, September 2007).

\section{In Vitro Configuration in a Pulse Duplicator}

Six ADVANTAGE size 23 aortic valves were fixed in a pulse duplicator, which allowed for high-speed imaging, ultrasound examination from the inflow aspect of the valve, or simultaneous recording of the flow meter signals with the electromagnetic flow meter probe in series to the valve. Flow meter signals were simultaneously recorded on the color Doppler images of the ultrasound system. The pulse duplicator stroke volume and frequency were adjusted until the IR phenomena were observed. It was also necessary to manipulate the valve orientation to produce the IR phenomenon. The valve was affixed in the hydraulic loop in 4 different orientations to gravity (Figure 1).

\section{In Vitro Color Doppler Flow Assessment}

The color Doppler images were recorded from the inflow side of the valve using an ultrasound system that is widely used in clinical practice (General Electric Vivid 7, Waukesha, Wis). This view is comparable to an in vivo apical view.

\section{High-Speed Imaging}

Images of the ADVANTAGE valve during a normal beat closure and an IR beat closure were obtained with a digital camera running at 1000 frames per second.

\section{RESULTS}

\section{Clinical Findings}

Since August 2003, echocardiographic assessment revealed IR in 18 reported cases in different centers. IR was detected in valve sizes 21 to 27 : valve size 21 in 2 cases, valve size 23 in 12 cases, valve size 25 in 2 cases, valve size 27 in 1 case, and valve size unknown in 1 case (Medtronic Inc, personal communication, September 2007).

Three reoperations were performed for suspected valve thrombosis. During 1 reoperation in a patient presenting with IR, the valve was left in place after exclusion of valve thrombosis; after the reoperation, the valve still showed IR. In another patient, the valve was explanted and replaced by a bioprosthesis. In the third patient, the ADVANTAGE valve showing IR was replaced with another ADVANTAGE valve, which then again showed IR.

\section{Color Doppler Assessment}

The video images from the in vitro and in vivo color Doppler examination are shown under normal operation (Figure 2, $A$ ) and in the case of IR (Figure 2, B). Color Doppler flow shows larger velocities and a larger color-coded area in IR. The schematic drawings illustrate the typical color Doppler findings from the valve's inflow side.

\section{High-Speed Imaging of Valve Closure}

Asynchronous closing of normal functioning bileaflet valves is a well-known and documented phenomenon. ${ }^{6}$ During normal closure, the first-to-close leaflet comes to a fully closed position. As the tip of the first-to-close leaflet makes contact with the housing major radius, the leading edge of the leaflet slides across the midline of the housing and stops moving. As the second-to-close leaflet moves toward closure, its leading edge makes contact with the leading edge of the first-to-close leaflet. After contact between the 2 leaflets, there is a normal rocking (sliding back-andforth) that results in the leading edge of the first-to-close leaflet being shifted back toward the midline. In vitro high-speed imaging showed that IR occurs when the shifting of the first-to-close leaflet back toward the midline is

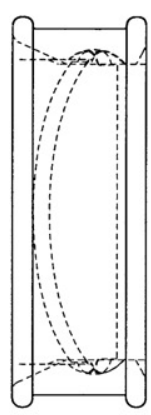

A

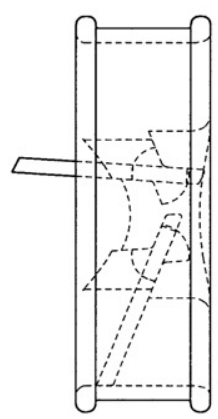

B

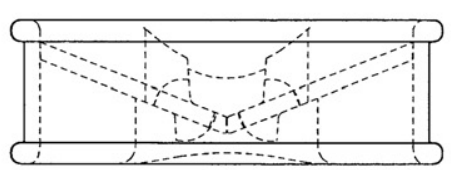

C

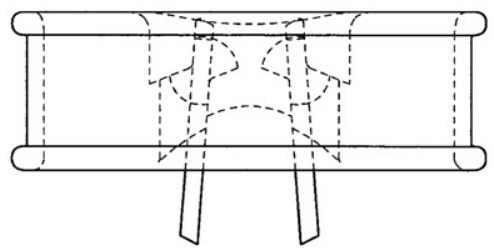

D

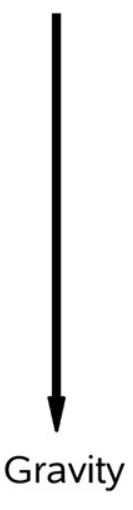

FIGURE 1. A, Horizontal valve axis with vertical leaflets, the rotational axis of the leaflets parallel to gravity. B, Horizontal valve axis with horizontal leaflets, the rotational axis of the leaflets perpendicular to gravity. C, D, Vertical valve axis with the inflow side down and up, the leaflets rotational axis perpendicular to gravity. 

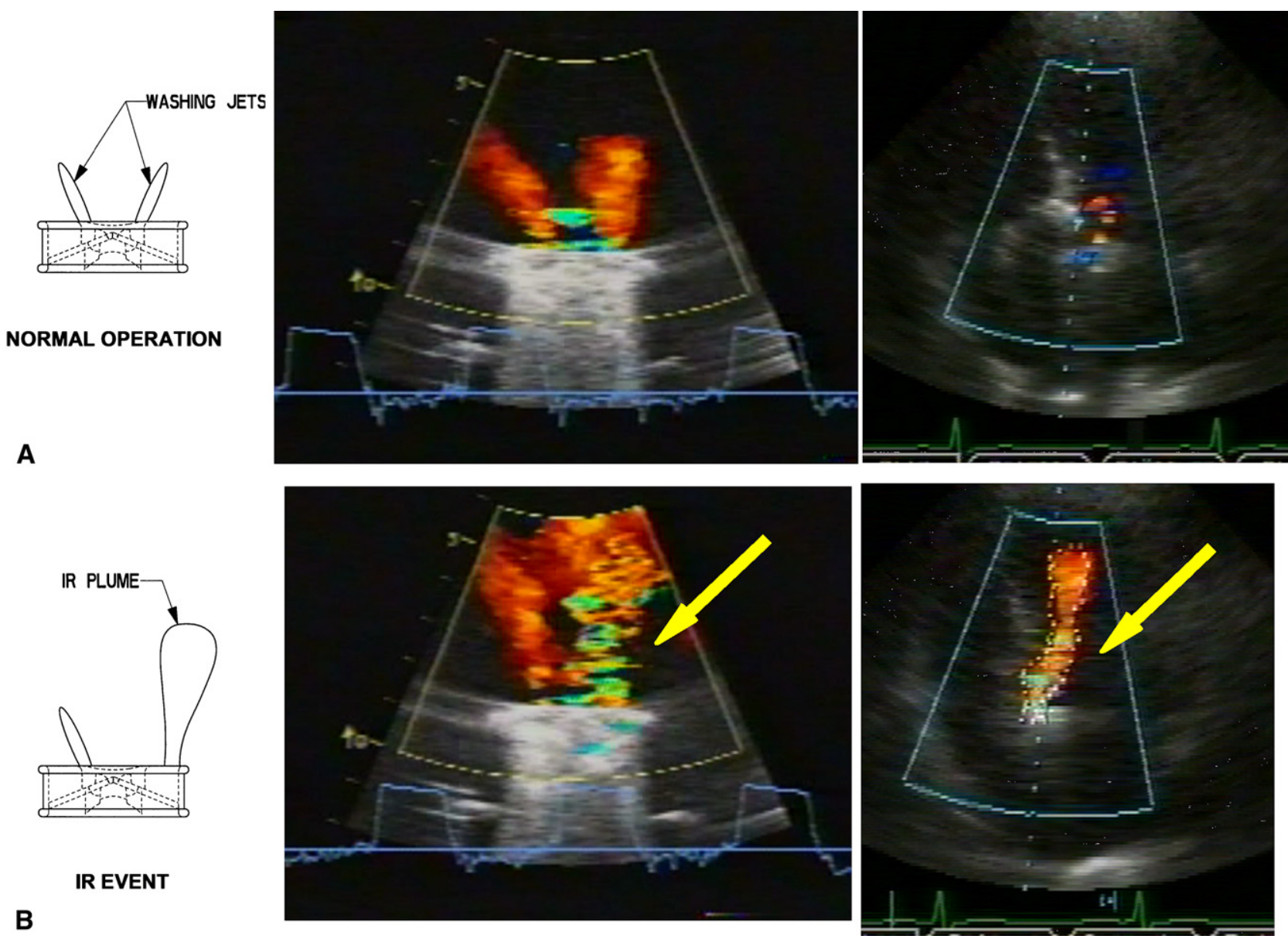

\section{B}

IR EVENT

FIGURE 2. A, Jets seen in a normal operation; in vitro echo image (center); in vivo echo with physiologic backflow from an apical view (right). B, Jets in an IR beat (left), IR in vitro echo image (center), in vivo echo from an apical view (right). Typical IR plume in color Doppler flow imaging (arrow). IR, Intermittent regurgitation.

incomplete. The leading edge of the second-to-close leaflet makes contact with the chamfer on the leading edge of the first-to-close leaflet, and the tip of the second-to-close leaflet is therefore prevented from coming to rest against the housing major radius. It stops short of the housing major radius, and a crescent-shaped gap exists between the leaflet tip and the housing major radius. Figure 3 illustrates the mechanism leading to an IR. A complete closure during diastole is shown in Figure 4, $A$, and an incomplete closure is shown in Figure 4, $B$.

\section{Hydrodynamic Measurements}

All 6 of the valves tested exhibited in vitro IR at 68 beats/ $\mathrm{min}$ and a cardiac output ranging from 4.0 to $4.5 \mathrm{~L} / \mathrm{min}$. One of the 6 valves was tested at 47 and 95 beats/min with cardiac output of 2.9 and $5.8 \mathrm{~L} / \mathrm{min}$, respectively. Under these conditions, IR was not detected. In the 7 study patients presenting with IR at the German Heart Center Munich, heart rates ranged from 45 to 95 beats/min and echocardiographically determined cardiac outputs ranged from 3.5 to $8.3 \mathrm{~L} /$ $\mathrm{min}$. In vitro, the regurgitant fraction can increase from a nor- mal of $6 \%$ to $25 \%$ during an IR beat. For the same valve, the in vitro IR regurgitant volume was not the same every time the IR event occurred. In vivo, the IR beat regurgitation was graded mild to moderate.

\section{Intermittent Regurgitation Sensitivity to Valve Orientation in the Gravitational Field}

In vitro, the leaflet rotational axis has to be in the horizontal orientation to reproduce the IR phenomenon. In this orientation, gravity maximizes asynchronous closure and works against the closure of the second-to-close leaflet. According to the different orientations of the valve in the gravitational field (Figure 1), the frequency of IR events observed varied between $0 \%$ and $6.4 \%$ (Figure $1, A, D, 0 \% ; B, 6.4 \% ; C, 5 \%$ ).

\section{Summary of the Results}

The in vitro findings showed that IR is caused by a physical interaction of the leaflets. IR has been replicated in vitro on every tested ADVANTAGE size 23 aortic valve. Several factors influencing IR have been elucidated during testing of 


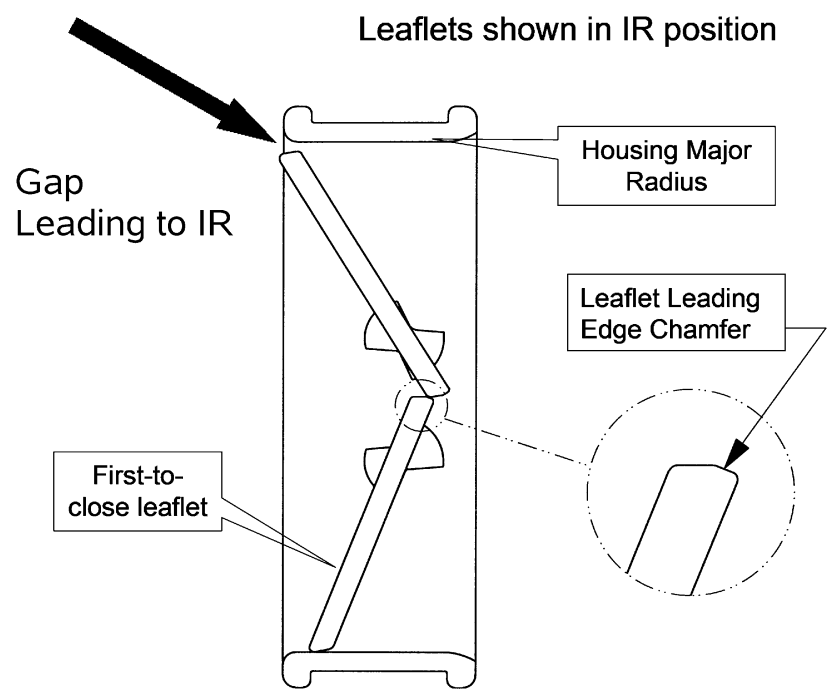

FIGURE 3. Leaflets shown in IR position. Gap leading to IR phenomenon (arrow). IR, Intermittent regurgitation.

the valves. The following observations have been made during the testing.

1. For IR to occur, the leaflet rotational axis has to be in the horizontal orientation, with the inflow aspect of the valve oriented either horizontal or down.

2. The frequency of in vitro IR with the size 23 valve varies from $0 \%$ to $6 \%$ of the cardiac cycles depending on the orientation of the valve to gravity.

3. The regurgitant fraction with the size 23 valve in vitro can increase from a normal of $6 \%$ to $25 \%$ during an IR beat.

4. In vitro IR has never resulted in failure of the leaflets to open.

\section{DISCUSSION}

There are many reports in the literature dealing with structural failures of mechanical heart valves affecting both mitral and aortic prostheses. ${ }^{7-9}$ These events are often life-threatening, and a detailed investigation is necessary if any abnormality is observed in the follow-up of a patient treated with a mechanical heart valve. Therefore, after the clinical detection and description of the IR phenomenon, ${ }^{1,2}$ an in vitro study was initiated to identify the underlying mechanism and to discern whether the valves that show IR are at an increased risk for structural failure.

IR could be reproduced in all 6 valves that were tested in the in vitro setting. All tested valves fulfilled the manufacturer's quality specifications. The contact of the leading edge of the second-to-close leaflet with the chamfer of the first-to-close leaflet was identified as the underlying mechanism. In vitro, the occurrence of the phenomenon was always intermittent. An every beat regurgitation could not be reproduced in the in vitro setting.

An every beat regurgitation case was published in November $2007^{10}$ : Nine weeks after aortic valve replacement with an ADVANTAGE prosthesis, the patient returned to the hospital with new-onset dyspnea. Echocardiographic evaluation showed an every beat aortic regurgitation grade II to III with 2 regurgitant jets. During reoperation, in situ testing showed that the valve insufficiency was caused by distinct leaflet interference in the valve hinge, which could be eliminated by applying gentle pressure to the leaflet. The valve was explanted and returned to the manufacturer for further investigation. On reexamination of the explant, the opening angle exceeded 90 degrees and a leaflet overlap was noticed. Because the valve was functioning normally in the early postoperative period, wear of the pivot areas was suspected as the underlying mechanism of valve failure by the authors. Whether there is any association to the mechanism causing IR remains unclear.

However, IR has led to 3 reoperations, of which 1 case has been published. ${ }^{3}$ This report describes the reoperation of a patient in which the IR phenomenon was misinterpreted as a thrombotic obstruction limiting leaflet excursions.
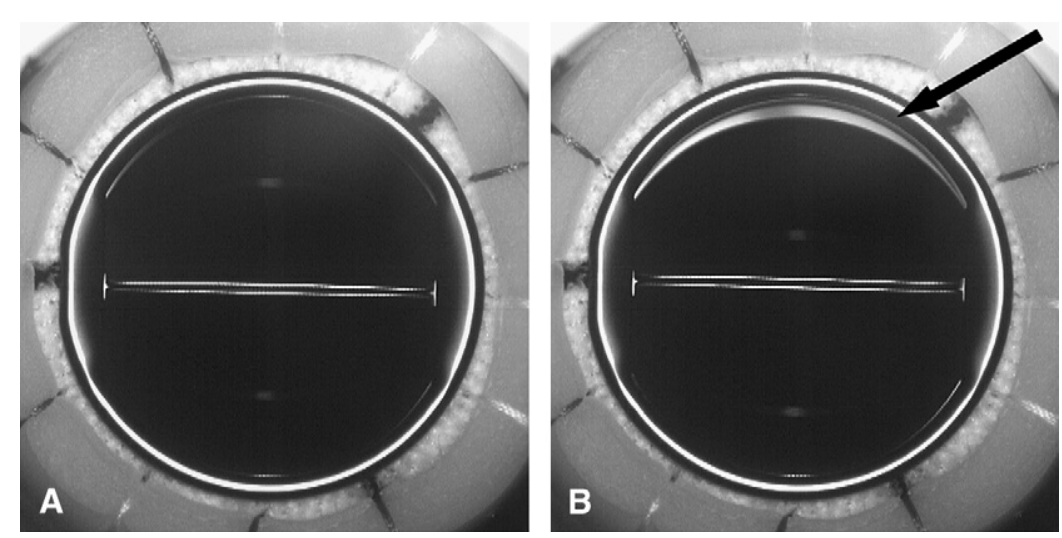

FIGURE 4. A, High-speed imaging of valve closure: Complete valve closure during diastole. B, Incomplete closure of the upper leaflet during an IR beat. Gap leading to IR phenomenon (arrow). 
During intraoperative inspection, the valve did not show any pathologic finding and was left in place. The patient showed an uneventful postoperative course, but the IR persisted after the reoperation. In 1 of the other 2 cases of reoperation, the ADVANTAGE valve was replaced with a bioprosthesis. In the third case of reoperation, the ADVANTAGE valve was replaced by another ADVANTAGE valve that then also showed IR. This suggests that patient-related factors may contribute to IR. Our assumed patient-related factors are individual flow and pressure conditions in the aortic root that prevent the first-to-close leaflet to slip back in a position that allows the second-to-close leaflet to reach the complete closing position. These special conditions in the aortic root may depend on the aortic geometry (angle between left ventricular outflow tract and ascending aorta, width of the ascending aorta, and sinuses of Valsalva), blood pressure, peripheral vessel resistance, heart rhythm and rate, and therefore length of diastole and end-diastolic left ventricular pressure.

IR in a mechanical valve has also been reported in cases of nonstructural valve deterioration. In 1 published case, involving another manufacturer's bileaflet mechanical valve, subvalvular pannus overgrowth on the inflow aspect of the prosthesis impeded normal closure of 1 leaflet causing severe aortic regurgitation, which occurred intermittently. ${ }^{11}$ This case demonstrates again the necessity of a precise identification of the mechanism causing IR.

The Medtronic ADVANTAGE was implanted in a human for the first time in 1999. To date, approximately 11,000 ADVANTAGE valves have been implanted in patients worldwide. To the present, 16 publications deal with the Medtronic ADVANTAGE prosthesis: Four studies provide in vitro data, ${ }^{4,12-14} 1$ animal study is presented, ${ }^{15} 1$ study focuses on mitral valve replacement, ${ }^{16}$ and 10 studies present human in vivo data concerning the aortic ADVANTAGE valve. ${ }^{1-3,10,17-22}$ The IR phenomenon is discussed in only 4 publications. ${ }^{1-3,10}$ This is partly because IR was unknown until the first report in March 2006. Because it is not likely that the IR phenomenon did not exist before 2006, this shows the difficulty in detecting an event that is characterized by its intermittent character. It is most likely that the standard echo protocol with documentation of color Doppler loops for only a few cardiac cycles underestimates the actual incidence of IR because of the intermittent character of the phenomenon. We hypothesize that the main issue for the detection of IR in vivo is to record long continuous echo loops. The echocardiographer also should be aware of the typical color flow pattern of an IR beat to not confuse it with the mitral valve inflow signal or misinterpret IR as a paravalvular leak or valve thrombosis with permanent obstruction of the leaflet's motion.

Two further publications report data for the ADVANTAGE Supra, a modified sewing ring design for complete supra-annular implantation. ${ }^{23,24}$ Within the ADVANTAGE Supra series, the IR phenomenon has not been reported. IR has also not been reported in mitral valves, possibly because mitral regurgitation in a mechanical valve is covered by the valve's shadow in a transthoracic apical view; thus, IR in the ADVANTAGE mitral valve cannot be completely excluded by means of transthoracic echo. Even the leakage volume through the hinge regions is often not clearly identified by transthoracic echocardiography. ${ }^{5}$ Alternately, the absence of reports of IR in the mitral position may be explained by the larger pressure differential across the mitral valve relative to the aortic valve. It is possible that the larger pressure differential provides a sufficient leaflet closing force to overcome an incomplete closure of a leaflet.

In other clinical studies concerning mechanical valves, ${ }^{25}$ transvalvular incompetence that exceeded the closing volume was diagnosed in $2 \%$ to $3 \%$ of studied valves. This observation did not lead to reoperation. The most common reasons for reoperation in mechanical valves are dehiscence, thrombosis, and pannus in-growth. ${ }^{26}$

However, 2 major questions remain to be clarified: (1) Is there any worsening of the long-term hemodynamic and clinical outcome in patients with ADVANTAGE valves exhibiting IR? (2) Can the IR phenomenon lead to a persistent dysfunction of valves in the sense of structural valve failure? In regard to the first question, the manufacturer has reviewed the records of patients with IR in the ADVANTAGE worldwide clinical study $(n=12)$. Their medical records did not indicate there were different adverse events occurring with this group, nor was there a higher adverse event rate occurring with this group than with the overall study cohort. In regard to the second question, it should be noted there have been no reports of leaflet or housing fractures during the 9-year clinical history of the valve. As clinical investigators for the ADVANTAGE valve, we implanted 84 valves in patients in the worldwide, multicenter trial. These patients continue to have satisfactory clinical outcomes as previously reported. ${ }^{2}$ We conclude that continued observation of patients with an ADVANTAGE valve in the aortic position is warranted, including consecutive echocardiograms to discern potential hemodynamic or volume loading consequences in the long term with this valve.

\section{CONCLUSIONS}

In vitro testing demonstrated IR in every size $23 \mathrm{AD}$ VANTAGE valve tested depending on cardiac output and the valve's orientation to gravity. Because IR has been reported in multiple ADVANTAGE valve sizes (21, 23, 25, and 27), we hypothesize that the mechanism underlying IR elucidated in this article has the potential to cause the IR phenomenon across the spectrum of ADVANTAGE valve sizes. There are no signs of structural abnormalities in the explanted valves showing IR (Medtronic personal communication). To avoid further unnecessary reoperations on the approximately 11,000 patients who have received ADVANTAGE valves worldwide, the phenomenon must be known 
in detail by all physicians who provide routine medical care for these patients.

\section{References}

1. Eichinger WB, Wagner I, Bleiziffer S, von Canal F, Lange R. Occasional singlebeat regurgitation observed with the new Medtronic ADVANTAGE bileaflet heart valve. J Thorac Cardiovasc Surg. 2006;131:730-1.

2. Eichinger WB, Wagner IM, Bleiziffer S, von Canal F, Gunzinger R, Ruzicka DJ, et al. Occasional single beat regurgitation observed with the Medtronic Advantage bileaflet heart valve. Ann Thorac Surg. 2006;82:537-41.

3. Haaverstad R, Vitale N, Tromsdal A, Hegbom K, Karevold A. Intermittent incomplete closure of Medtronic Advantage aortic valve leaflets causes unnecessary reoperation. J Thorac Cardiovasc Surg. 2007;134:791-2.

4. Saxena R, Lemmon J, Ellis J, Yoganathan A. An in vitro assessment by means of laser Doppler velocimetry of the Medtronic Advantage bileaflet mechanical heart valve hinge flow. J Thorac Cardiovasc Surg. 2003;126:90-8.

5. Chambers J, Cross J, Deverall P, Sowton E. Echocardiographic description of the CarboMedics bileaflet prosthetic heart valve. I Am Coll Cardiol. 1993;21: 398-405.

6. Donnerstein RL, Scott WA, Vasu A, Copeland JG. Acoustic analysis of the closing sounds of bileaflet prosthetic valves in a sheep model. J Thorac Cardiovasc Surg. 1991;101:1060-8.

7. Bottio T, Casarotto D, Thiene G, Caprili L, Angelini A, Gerosa G. Leaflet escape in a new bileaflet mechanical valve: TRI technologies. Circulation. 2003;107: 2303-6.

8. Mosterd A, Shahin GM, van Boven WJ, Jaarsma W, Graafland AD, van Swieten HA. Images in cardiovascular medicine. Leaflet fracture of a St. Jude mechanical bileaflet valve. Circulation. 2005;111:e280-1.

9. Blot WJ, Ibrahim MA, Ivey TD, Acheson DE, Brookmeyer R, Weyman A, et al. Twenty-five-year experience with the Bjork-Shiley convexoconcave heart valve: a continuing clinical concern. Circulation. 2005;111:2850-7.

10. Hansen L, Danne M, Hoffmann B, Riess FC. Structural valve failure with every beat regurgitation observed using the Medtronic Advantage aortic valve. J Thorac Cardiovasc Surg. 2007;134:1344-5.

11. Galli CA, Muratori M, Montorsi P, Barili F, Polvani G, Pepi M. Cyclic intermittent aortic regurgitation of a mechanical bileaflet aortic valve prosthesis: diagnosis and clinical implications. $J$ Am Soc Echocardiogr. 2007;20:1315. e5-1315.e8.

12. Shu MC, Gross JM, O'Rourke KK, Yoganathan AP. An integrated macro/micro approach to evaluating pivot flow within the Medtronic ADVANTAGE bileaflet mechanical heart valve. J Heart Valve Dis. 2003;12:503-12.

13. Shu MC, O'Rourke KK, Coppin CM, Lemmon JD. Flow characterization of the ADVANTAGE and St. Jude Medical bileaflet mechanical heart valves. J Heart Valve Dis. 2004;13:814-22.
14. Rakow N, Nelson D, McClay C, Rose R, Leuer L, Boldt C, et al. Chronic, preclinical assessment of the Medtronic Advantage aortic valve prosthesis. J Heart Valve Dis. 2002;11:851-6.

15. Kleine P, Scherer M, Abdel-Rahman U, Klesius AA, Ackermann H, Moritz A. Effect of mechanical aortic valve orientation on coronary artery flow: comparison of tilting disc versus bileaflet prostheses in pigs. J Thorac Cardiovasc Surg. 2002; 124:925-32.

16. Reiss N, Seifert D, Koertke H, Hoffmann-Koch A, Kleikamp G, Bairaktaris A, et al. Initial experience with the medtronic ADVANTAGE valve prosthesis in the mitral position-clinical outcome and analysis of complications. Thorac Cardiovasc Surg. 2007;55:149-55.

17. Bakhtiary F, Abolmaali N, Dzemali O, Wittlinger T, Doss M, Moritz A, et al. Impact of mechanical and biological aortic valve replacement on coronary perfusion: a prospective, randomized study. J Heart Valve Dis. 2006;15:5-11.

18. Guenzinger R, Eichinger WB, Botzenhardt F, Bleiziffer S, Wagner I, Bauernschmitt R, et al. Rest and exercise performance of the Medtronic Advantage bileaflet valve in the aortic position. Ann Thorac Surg. 2005;80:1319-26.

19. Haaverstad R, Vitale N, Karevold A, Cappabianca G, Tromsdal A, Olsen PS, et al. Clinical and echocardiographic assessment of the Medtronic Advantage aortic valve prosthesis: the Scandinavian multicentre, prospective study. Heart. 2007; 93:500-5.

20. Kleine P, Klesius AA, Scherer M, Abdel-Rahman U, Moritz A. Initial in vivo results of the new Medtronic Advantage(TM) bileaflet valve in aortic position and comparison to the SJM. Cardiovasc Surg. 2002;10:494-9.

21. Koertke H, Seifert D, Drewek-Platena S, Koerfer R. Hemodynamic performance of the Medtronic ADVANTAGE prosthetic heart valve in the aortic position: echocardiographic evaluation at one year. J Heart Valve Dis. 2003;12:348-53.

22. Korfer R, Svennevig JL, Mohr FW, Leguerrier A, Menicanti L, Olsen PS, et al. The worldwide mid-term experience with the Medtronic ADVANTAGE bileaflet mechanical heart valve. J Heart Valve Dis. 2006;15:404-13.

23. Guenzinger R, Eichinger WB, Botzenhardt F, Bleiziffer S, Wagner I, Bauernschmitt R, et al. A prospective randomized comparison of the Medtronic Advantage Supra and St. Jude Medical Regent mechanical heart valves in the aortic position: is there an additional benefit of supra-annular valve positioning? J Thorac Cardiovasc Surg. 2008;136:462-71. Epub 2008 May 12.

24. Bottio T, Tarzia V, Rizzoli G, Gerosa G. In vitro testing of three totally supra-annular bileaflet mechanical valves: hydrodynamics in the Sheffield pulse duplicator. J Heart Valve Dis. 2008;17:222-6.

25. Autschbach R, Walther T, Falk V, Kostelka M, Rosler A, Langebartels G, et al. Prospectively randomized comparison of different mechanical aortic valves. Circulation. 2000;102(19 Suppl. 3):III1-4.

26. Rizzoli G, Guglielmi C, Toscano G, Pistorio V, Vendramin I, Bottio T, et al. Reoperations for acute prosthetic thrombosis and pannus: an assessment of rates, relationship and risk. Eur J Cardiothorac Surg. 1999;16:74-80. 